\title{
THE MACKEY BIJECTION FOR COMPLEX REDUCTIVE GROUPS AND CONTINUOUS FIELDS OF REDUCED GROUP C*-ALGEBRAS
}

\author{
NIGEL HIGSON AND ANGEL ROMÁN
}

\begin{abstract}
The purpose of this paper is to make a further contribution to the Mackey bijection for a complex reductive group $G$, between the tempered dual of $G$ and the unitary dual of the associated Cartan motion group. We shall construct an embedding of the $C^{*}$-algebra of the motion group into the reduced $C^{*}$-algebra of $G$, and use it to characterize the continuous field of reduced group $C^{*}$-algebras that is associated to the Mackey bijection. We shall also obtain a new characterization of the Mackey bijection using the same embedding.
\end{abstract}

\section{INTRODUCTION}

The Mackey bijection is a certain one-to-one correspondence between the tempered irreducible unitary representations of a real reductive group and the unitary irreducible representations of its Cartan motion group. Its existence was suggested by George Mackey [Mac75] in the 1970's (although Mackey stopped short of making a precise conjecture). After a long pause, Mackey's proposal began to be examined in detail over the past dozen years, first for complex groups in Hig08 and ultimately for all real groups in breakthrough work of Afgoustidis Afg15.

The purpose of this paper is to return to the case of complex groups, and examine the Mackey bijection in greater detail through the theory of group $C^{*}$-algebras and continuous fields of group $C^{*}$-algebras. We shall characterize the continuous field that has played a key role in nearly every study of the Mackey bijection, and use our characterization of the field to give a new characterization of the Mackey bijection.

Group $C^{*}$-algebras have long played a prominent role in the Mackey bijection. Indeed Mackey's original idea was kept alive by Alain Connes, who noticed a link between Mackey's work and the Connes-Kasparov isomorphism in $C^{*}$-algebra $K$ theory CH90, BCH94.

The connection with $K$-theory is made through the deformation to the normal cone construction from geometry, which associates to the inclusion of a maximal compact subgroup $K$ into any Lie group $G$ a smooth, one-parameter family of Lie groups $G_{t}$, all of them copies of the group $G$ except at $t=0$, where

$$
G_{0}=K \ltimes(\operatorname{Lie}(G) / \operatorname{Lie}(K)) .
$$

In the case where $G$ is reductive, $G_{0}$ is the Cartan motion group. Associated to the deformation to the normal cone construction there is a continuous field of $C^{*}$-algebras $\left\{C_{r}^{*}\left(G_{t}\right)\right\}_{t \in \mathbb{R}}$. It turns out that in the reductive case this continuous

Received by the editors November 3, 2019, and, in revised form, June 19, 2020.

2020 Mathematics Subject Classification. Primary 22E45, 46L99. 
field is assembled from constant fields of $C^{*}$-algebras (indeed commutative $C^{*}$ algebras) by natural operations: extensions, Morita equivalences and direct limits. This immediately proves the Connes-Kasparov isomorphism, and by making the assembly process explicit we obtain an explicit Mackey bijection. For details see Hig08 and Afg16.

To repeat what we wrote earlier, a principal goal of this paper is to study the continuous field $\left\{C_{r}^{*}\left(G_{t}\right)\right\}_{t \in \mathbb{R}}$ in more detail, and indeed to characterize it up to isomorphism in the case where $G$ is a complex reductive group. To explain the method, it is helpful to start with a toy model case, in which $G$ is a semidirect product group

$$
G=K \ltimes V
$$

associated to the action of a compact group $K$ on a real, finite-dimensional vector space $V$ (this is not a reductive group, of course). Here the deformation to the normal cone associated to the embedding of $K$ into $G$ gives a smooth family of groups $\left\{G_{t}\right\}$ that is isomorphic to the constant family of groups with fiber $G$. However $\left\{G_{t}\right\}$ is not equal to the constant family; to obtain an isomorphism to the constant family we must use the family of rescaling morphisms

$$
\begin{gathered}
\alpha_{t}: G \longrightarrow G \\
\alpha_{t}(k, v)=(k, t v)
\end{gathered}
$$

for $t \neq 0$. Applying $\alpha_{t}$ to the fiber at $t \neq 0$, and the identity morphism at $t=0$, we obtain an isomorphism from the constant family of groups with fiber $G$ into the deformation to the normal cone family. The same rescaling morphisms induce an isomorphism from the constant field of $C^{*}$-algebras with fiber $C_{r}^{*}(G)$ to the deformation to the normal cone continuous field $\left\{C_{r}^{*}\left(G_{t}\right)\right\}$.

Similar rescaling morphisms do not exist at the group level on a real reductive group, except in trivial cases. But one might ask whether they exist nonetheless on the reduced group $C^{*}$-algebra?

The reason that one might guess that rescaling morphisms exist at the $C^{*}$-algebra level is that the structure of the $C^{*}$-algebra is very closely tied to the structure of the tempered dual of $G$, and there is a natural rescaling operation on the tempered dual. Indeed the tempered dual is parametrized by a combination of discrete and continuous parameters, with the latter belonging to vector spaces, or quotients of vectors spaces by finite group actions. So the continuous parameters may be rescaled in the obvious way. Moreover this rescaling operation plays a central role in the Mackey bijection.

Our first main result is that rescaling morphisms for connected complex reductive groups do indeed exist at the $C^{*}$-algebra level:

Theorem. Let $G$ be a connected complex reductive group. There is a one-parameter group of automorphisms

$$
\alpha_{t}: C_{r}^{*}(G) \longrightarrow C_{r}^{*}(G) \quad(t>0)
$$

that implements the rescaling action on the tempered dual of $G$ in the Mackey bijection. 
See Section 4. Our second main result is that, at the level of continuous fields, there is a (unique) extension to $t=0$, as follows:

Theorem. Let $G$ be a connected complex reductive group and let $\left\{f_{t}\right\}$ be a continuous section of the continuous field of $C^{*}$-algebras associated to the deformation to the normal cone construction for the inclusion of a maximal compact subgroup into $G$. Then the limit $\lim _{t \rightarrow 0} \alpha_{t}\left(f_{t}\right)$ exists in $C_{r}^{*}(G)$, and the formula

$$
\alpha\left(f_{0}\right)=\lim _{t \rightarrow 0} \alpha_{t}\left(f_{t}\right)
$$

defines an embedding of $C^{*}$-algebras

$$
\alpha: C_{r}^{*}\left(G_{0}\right) \longrightarrow C_{r}^{*}(G) .
$$

See Theorems 5.1.1 and 5.2.2 for the precise statements (we have omitted here some details related to the Haar measures on the groups $G_{t}$, which vary with $t$ ).

Now, given an embedding of $C^{*}$-algebras $\alpha: B \rightarrow A$, there is a simple and obvious way to construct a continuous field of $C^{*}$-algebras with fibers

$$
A_{t}= \begin{cases}A & t \neq 0 \\ B & t=0\end{cases}
$$

namely we take as continuous sections all those set-theoretic sections for which the formula

$$
t \longmapsto \begin{cases}a_{t} & t \neq 0 \\ \alpha\left(b_{0}\right) & t=0\end{cases}
$$

defines a continuous function from $\mathbb{R}$ to $A$. Let us call this the mapping cone continuous field associated to the inclusion. Using mapping cones, we are able to characterize the continuous field associated to the deformation to the normal cone, as follows:

Theorem. Let $G$ be a connected complex reductive group. The continuous field of $C^{*}$-algebras $\left\{C_{r}^{*}\left(G_{t}\right)\right\}_{t \in \mathbb{R}}$ associated to the deformation to the normal cone construction is isomorphic to the mapping cone field for the embedding

$$
\alpha: C_{r}^{*}\left(G_{0}\right) \longrightarrow C_{r}^{*}(G) \text {. }
$$

Indeed the morphism

$$
\left\{f_{t}\right\} \longmapsto\left\{\alpha_{t}\left(f_{t}\right)\right\}
$$

where $\alpha_{0}=\mathrm{id}$, is a bijection from continuous sections of the deformation to the normal cone field to continuous sections of the mapping cone field.

See Section 5.3 for further details (including the proper treatment of $\alpha_{t}$ when $t$ is negative).

To summarize, one might say that the continuous field is nothing more or less than the morphism

$$
\alpha: C_{r}^{*}\left(G_{0}\right) \longrightarrow C_{r}^{*}(G) .
$$

What does this tell us about the Mackey bijection? Each tempered irreducible unitary representation of $G$ corresponds to an irreducible representation

$$
\pi: C_{r}^{*}(G) \longrightarrow \mathfrak{B}\left(H_{\pi}\right)
$$

(in fact the range is the ideal of compact operators, $\mathfrak{K}\left(H_{\pi}\right)$, but that is not relevant here). The composition of this representation of $C_{r}^{*}(G)$ with the embedding $\alpha$ above is not necessarily irreducible, so composition with $\alpha$ does not directly determine a 
map from the tempered dual of $G$ to the unitary dual of $G_{0}$. However generically the restriction is irreducible, and it turns out that this enough to determine a unique Mackey bijection:

Theorem. Let $G$ be a connected complex reductive group. There is a unique bijection $\mu$ from the tempered unitary dual of $G$ to the unitary dual of $G_{0}$ with the property that for every $\pi \in \widehat{G}_{r}, \mu(\pi)$ is realizable as a subrepresentation of $\pi \circ \alpha$.

See Section 6. It is an outstanding problem to provide some sort of conceptual explanation for the Mackey bijection, and we do not know if the approach in this paper will contribute usefully to the solution. At the moment, it seems to us that progress in this direction will depend on understanding the rescaling morphisms $\alpha_{t}$, or perhaps their infinitesimal generator, more conceptually. Given the way that the rescaling morphisms are defined (see Section 4) that would, in turn, seem to depend on developing some kind of understanding of the relation between the Mackey bijection and the Plancherel measure, which enters into the formula for the inverse of the Fourier transform isomorphism for $G$.

As for the problem of extending the approach of this paper to real groups, the major issue is to understand the (normalized) intertwining isomorphisms between unitary principal series representations in much greater detail (and thanks to the work of Harish-Chandra, and Knapp and Stein, this is in turn related to the theory of the Plancherel measure). But there appear to be no obstructions, beyond this difficulty, in handling the case of real groups. An encouraging indication of this is the case of $S L(2, \mathbb{R})$, which is studied in Rom19.

Finally, there are very interesting similarities between the results of this paper and results in the representation theory of $p$-adic groups, and especially in the tempered representation theory of affine Hecke algebras. See [Sol12], where scaling morphisms are defined and studied on the associated reduced $C^{*}$-algebras, and a Mackey-type bijection of representations is established. See also [BK18].

\section{Continuous fields of Reduced group $\mathrm{C}^{*}$-Algebras}

2.1. Deformation spaces. There is a deformation space or deformation to the normal cone $\mathbb{N}_{V} M$ associated to the embedding of any smooth manifold $M$ as a closed submanifold of a smooth manifold $V$. It is a smooth manifold itself, and it is equipped with a submersion onto the real line. See for example Hig08, Sec. 6] for an account adapted to the concerns of this paper, although the definition originates elsewhere and much earlier, in algebraic geometry (see [Ful84, Ch. 5] for the algebraic-geometric perspective).

Let us quickly review the construction in the case of interest to us, which is the inclusion of a maximal compact subgroup $K$ into an almost connected Lie group $G$. As a set, the deformation space is defined to be

$$
\mathbb{N}_{G} K=N_{G} K \times\{0\} \sqcup \bigsqcup_{t \neq 0} G \times\{t\},
$$

where $N_{G} K$ is the normal bundle to $K$,

$$
N_{G} K=\left.T G\right|_{K} / T K .
$$

The deformation space has a unique smooth manifold structure for which

(i) The natural map $N_{G} K \rightarrow \mathbb{R}$ is smooth. 
(ii) If $f$ is a smooth function on $G$, then the function

$$
\left\{\begin{array}{l}
(g, t) \mapsto f(g) \quad t \neq 0 \\
\left(X_{k}, 0\right) \mapsto f(k)
\end{array}\right.
$$

is smooth on $\mathbb{N}_{G} K$.

(iii) If $f$ is a smooth function on $G$, and if $f$ vanishes on $K$, then the function

$$
\left\{\begin{array}{l}
(g, t) \mapsto t^{-1} f(g) \quad t \neq 0 \\
\left(X_{k}, 0\right) \mapsto X_{k}(f)
\end{array}\right.
$$

is smooth on $\mathbb{N}_{G} K$.

(iv) At every point, local coordinates can be selected from functions of the above types.

The commuting diagram of multiplication operations

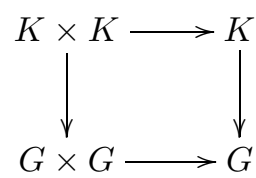

induces a diagram

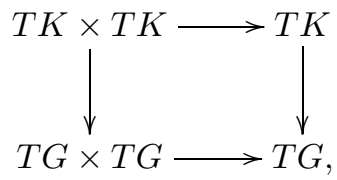

and we obtain from this a multiplication operation

$$
N_{G} K \times N_{G} K \longrightarrow N_{G} K .
$$

It makes the normal bundle into a Lie group, and we obtain from $\mathbb{N}_{G} K$ a smooth family of Lie groups over $\mathbb{R}$ (that is, a Lie groupoid with object space $\mathbb{R}$ and source and target maps equal to one another).

If we trivialize the tangent bundles on $G$ and $K$ by left translations, then we obtain an identification of bundles and Lie groups

$$
N_{G} K \cong K \ltimes \mathfrak{g} / \mathfrak{k}
$$

where on the right is the semidirect product group associated to the adjoint action.

2.2. The associated continuous field of $\mathbf{C}^{*}$-algebras. For $t \in \mathbb{R}$ let us denote by $G_{t}$ the fiber over $t$ of the submersion

$$
\mathbb{N}_{G} K \longrightarrow \mathbb{R}
$$

It is a Lie group, and for $t \neq 0$ it is isomorphic to $G$ itself. Choose a Haar measure $\mu$ for $G$. Then equip the fibers $G_{t}$ for $t \neq 0$ with the left Haar measure $d g_{t}=|t|^{-d} d g$, where

$$
d=\operatorname{dim}(G / K)
$$

(we shall use this notation throughout the paper). The Haar measure on $G$ determines an associated Haar measure $d g_{0}$ on $G_{0}$, and altogether the Haar measures that we have chosen for the fibers $G_{t}$ vary smoothly with $t$, in the sense that if $f$ is a 
smooth and compactly supported function on $\mathbb{N}_{G} K$, and if $f_{t}$ denotes the restriction of $f$ to $G_{t}$, then the integral

$$
\int_{G_{t}} f_{t}\left(g_{t}\right) d g_{t}
$$

is a smooth function of $t \in \mathbb{R}$. Moreover if $f_{1}$ and $f_{2}$ are two smooth and compactly supported functions on $\mathbb{N}_{G} K$, then their convolution product, defined fiberwise by

$$
\left(f_{1, t} * f_{2, t}\right)\left(g_{t}\right)=\int_{G_{t}} f_{1, t}\left(\gamma_{t}\right) f_{2, t}\left(\gamma_{t}^{-1} g_{t}\right) d \gamma_{t} \quad\left(g_{t} \in G_{t}\right),
$$

is a smooth and compactly supported function $\mathbb{N}_{G} K$, too.

The same convolution formula applied to functions on $G_{t}$ alone defines a product on $C_{c}^{\infty}\left(G_{t}\right)$, and we shall denote by $C_{r}^{*}\left(G_{t}\right)$ the reduced $C^{*}$-algebra completion (in the norm that $C_{c}^{\infty}\left(G_{t}\right)$ obtains as left-convolution operators on $L^{2}\left(G_{t}, d g_{t}\right)$, and the adjoint operation it obtains from the operator adjoint operation). Compare [Ped79, Sec. 7.2].

Lemma 2.2.2 (See Hig08, Lemma 6.13]). Let $G$ be an almost-connected Lie group and let $K$ be a maximal compact subgroup of $G$. If $f$ is a smooth and compactly supported function on the deformation space $\mathbb{N}_{K} G$, and if $f_{t}$ denotes its restriction to $G_{t}$, then the norm $\left\|f_{t}\right\|_{C_{r}^{*}\left(G_{t}\right)}$ is a continuous function of $t \in \mathbb{R}$.

It follows that the smooth and compactly supported functions on $\mathbb{N}_{G} K$ generate the continuous sections of a continuous field of $C^{*}$-algebras over $\mathbb{R}$ with fibers $C_{r}^{*}\left(G_{t}\right)$ in the sense of Dix77, Prop. 10.2.3]. We shall call this the deformation field of reduced group $C^{*}$-algebras associated to the inclusion of $K$ into $G$.

It will be helpful to think of the $C^{*}$-algebras $C_{r}^{*}\left(G_{t}\right)$ as being completely distinct from one another, even though for $t \neq 0$ they may be viewed as completions of the same space of functions in equivalent norms. When we wish to compare $C_{r}^{*}\left(G_{t}\right)$ for different $t \neq 0$, as we shall in Section 5, we shall do so using the canonical $*-$ isomorphisms

$$
\lambda_{t}: C_{r}^{*}\left(G_{t}\right) \stackrel{\cong}{\longrightarrow} C_{r}^{*}(G)
$$

associated to the isomorphisms $G_{t} \cong G$. These are given by the formulas

$$
\lambda_{t}: f_{t} \longmapsto\left[g \mapsto|t|^{-d} f_{t}(g)\right]
$$

for $f_{t} \in C_{c}^{\infty}\left(G_{t}\right)$. The factor $|t|^{-d}$ accounts for the change in Haar measures.

The isomorphisms $\lambda_{t}$ may also be described as follows. For $t \neq 0$ the left translation action of $G_{t} \cong G$ on itself integrates to a $C^{*}$-algebra representation

$$
\lambda_{t}: C_{r}^{*}\left(G_{t}\right) \longrightarrow \mathfrak{B}\left(L^{2}(G, d g)\right) .
$$

The image is independent of $t$, and is the image of the regular representation of $C_{r}^{*}(G)$ itself. So we obtain $*$-isomorphisms as above.

2.3. The case of reductive groups. If $G$ is a real reductive group, then the deformation space $\mathbb{N}_{G} K$ may be given a more concrete form using the following basic structural facts about $G$. Fix a Cartan decomposition $\mathfrak{g}=\mathfrak{k} \oplus \mathfrak{s}$ for the Lie algebra of $G$. Then of course $\mathfrak{g} / \mathfrak{k} \cong \mathfrak{s}$ and

$$
K \ltimes \mathfrak{g} / \mathfrak{k} \cong K \ltimes \mathfrak{s} .
$$

Next, choose a maximal abelian subspace of $\mathfrak{a} \subseteq \mathfrak{s}$ and an Iwasawa decomposition

$$
G=K A N
$$


where $A=\exp [\mathfrak{a}]$ (any two Iwasawa decompositions are conjugate by an element of $K)$. The smooth map

$$
\begin{aligned}
K \times \mathfrak{a} \times \mathfrak{n} \longrightarrow G \\
(k, X, Y) \longmapsto k \exp (X) \exp (Y)
\end{aligned}
$$

is then a diffeomorphism. See for example Kna02 for all this.

If we view $K$ as a submanifold of $K \times \mathfrak{a} \times \mathfrak{n}$ via the inclusion

$$
\begin{gathered}
K \longrightarrow K \times \mathfrak{a} \times \mathfrak{n} \\
k \longmapsto(k, 0,0),
\end{gathered}
$$

then the functoriality of the deformation space construction (see for example [HSSH18, p.303]) and the commutativity of the diagram

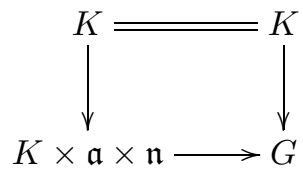

allows us to view $\mathbb{N}_{G} K$ as the deformation space associated to the inclusion of the zero section into a (trivial) vector bundle. For the latter, see Hig10, Ex. 4.6]. We obtain a diffeomorphism

$$
K \times \mathfrak{a} \times \mathfrak{n} \times \mathbb{R} \longrightarrow \mathbb{N}_{G} K
$$

given by the formula

$$
(k, X, Y, t) \longrightarrow \begin{cases}(k \exp (t X) \exp (t Y), t) & t \neq 0 \\ ((k,[X+Y]), 0) & t=0 .\end{cases}
$$

Here $[X+Y]$ denotes the class in the quotient vector space $\mathfrak{g} / \mathfrak{k}$ associated to the vector $X+Y \in \mathfrak{g}$, or equivalently, keeping in mind the isomorphism (2.3.1), the projection of $X+Y \in \mathfrak{a} \oplus \mathfrak{n}$ onto the summand $\mathfrak{s}$ in the Cartan decomposition $\mathfrak{g}=\mathfrak{k} \oplus \mathfrak{s}$.

This computation has the following consequence that will be of central importance later on:

Lemma 2.3.2. A function $f: \mathbb{N}_{G} K \rightarrow \mathbb{C}$ is smooth and compactly supported if and only if the function

$$
\tilde{f}:(k, X, Y, t) \longmapsto \begin{cases}f(k \exp (t X) \exp (t Y), t) & t \neq 0 \\ f(k,[X+Y]), 0) & t=0\end{cases}
$$

is smooth and compactly supported on $K \times \mathfrak{a} \times \mathfrak{n} \times \mathbb{R}$.

\section{Structure of Reduced group C*-Algebras}

The main purpose of this section is to review the detailed description of the $C^{*}$ algebra $C_{r}^{*}(G)$, which was presented first in PP83. This we shall use throughout the paper. We shall also review the classification of the irreducible unitary representations of the motion group, due to Mackey [Mac49], which we shall use in the final section. For completeness we shall also review the structure of $C_{r}^{*}\left(G_{0}\right)$; this will not play a significant role in what follows, but among other things the unitary dual of $G_{0}$ is most easily determined from the structure of $C_{r}^{*}\left(G_{0}\right)$. 
3.1. Connected complex reductive groups. Let $G$ be a complex, connected, reductive group. To describe the tempered unitary dual and reduced group $C^{*}$ algebra of $G$, we begin with an Iwasawa decomposition $G=K A N$. Let $M$ be the centralizer in $K$ of $A$, which is a maximal torus in $K$, and let $P$ be the (Borel or minimal parabolic) subgroup $M A N$. The group $N$ is a normal subgroup of $P$, and as a result of this, if $\sigma$ is a character of $M$, and if $\nu \in \mathfrak{a}^{*}$, then the formula

$$
\sigma \otimes \exp (i \nu): m \cdot \exp (X) \cdot n \longmapsto \sigma(m) \cdot \exp (i \nu(X))
$$

defines a unitary character of $P$. The unitary principal series representation of $G$ associated to $\sigma$ and $\nu$ (and the given choice of minimal parabolic subgroup) is the unitarily induced representation

$$
\pi_{\sigma, \nu}=\operatorname{Ind}_{P}^{G} \sigma \otimes \exp (i \nu) .
$$

By definition, the Hilbert space of this representation is the completion of the space of all smooth functions $\psi: G \rightarrow \mathbb{C}$ such that

$$
\psi(g m \exp (X) n)=e^{-(\rho+i \nu)(X)} \sigma(m)^{-1} \psi(g)
$$

for all $g \in G, m \in M, X \in \mathfrak{a}$ and $n \in N$, in the norm associated to the inner product

$$
\langle\phi, \psi\rangle=\int_{K} \overline{\phi(k)} \psi(k) d k
$$

(thanks to the relation (3.1.1) and the Iwasawa decomposition, the functions $\phi$ and $\psi$ are determined by their restrictions to $K)$. Here $\rho \in \mathfrak{a}^{*}$ is the half-sum of the positive restricted roots associated to our choice of $N$ (its presence makes the representation $\pi_{\sigma, \nu}$ unitary; other than that we shall not need any further information about it). The action of $G$ on the functions (3.1.1) is by left translation.

The Hilbert space just defined identifies, via restriction to $K$, with the Hilbert space

$$
L^{2}(K)^{\sigma}=\left\{\psi: K \stackrel{L^{2}}{\rightarrow} \mathbb{C}: \psi(k m)=\sigma(m)^{-1} \psi(k) \quad \forall m \in M, \forall k \in K\right\} .
$$

We shall work exclusively with this realization of the principal series from now on. An advantage in doing so is that the Hilbert space for the principal series $\pi_{\sigma, \nu}$ now depends only on $\sigma$, and not on $\nu$. A disadvantage is that the formula for the action of $G$ is a bit more complicated. To describe it we need to use the Iwasawa decomposition of elements of $G$ :

$$
g=\kappa(g) \cdot e^{H(g)} \cdot n,
$$

where $\kappa(g) \in K, H(g) \in \mathfrak{a}$, and $n \in N$. With this notation,

$$
\left(\pi_{\sigma, \nu}(g) \phi\right)(k)=e^{-(\rho+i \nu) H\left(g^{-1} k\right)} \phi\left(\kappa\left(g^{-1} k\right)\right)
$$

for $\phi \in L^{2}(K)^{\sigma}$. We shall use this formula in Section 5 .

The Weyl group of $K$,

$$
W=N_{K}(M) / M
$$

acts by conjugation on $M$, of course, but it also acts by conjugation on $A$ (this is because the complexification of $M$ is $M A$ ). So $W$ acts on the parameter space $\widehat{M} \times \mathfrak{a}^{*}$ for the unitary principal series. The main facts about the unitary principal 
series of $G$ may then be summarized as follows:

Theorem 3.1.5. Let $G$ be a connected, complex reductive group.

(i) Every unitary principal series representation is an irreducible, tempered unitary representation of $G$

(ii) Every irreducible, tempered unitary representation of $G$ is equivalent to some unitary principal series representation.

(iii) Two unitary principal series representations $\pi_{\sigma^{\prime}, \nu^{\prime}}$ and $\pi_{\sigma^{\prime \prime}, \nu^{\prime \prime}}$ are equivalent if and only if the pairs $\left(\sigma^{\prime}, \nu^{\prime}\right),\left(\sigma^{\prime \prime}, \nu^{\prime \prime}\right) \in \widehat{M} \times \mathfrak{a}^{*}$ are conjugate by an element of $W$.

These facts are essentially due to Gelfand and Naimark when $G$ is a classical group [GfN50], and to Harish-Chandra [HC54] in general, although the irreducibility of the unitary principal series in full generality is due to Wallach Wal71. (The question of whether or not the unitary principal series defines a closed subset of the unitary dual, and hence accounts for the full tempered dual, was not initially addressed. But see for example [CCH16] for this issue.)

Definition 3.1.6. Let $\sigma \in \widehat{M}$ and let $\nu \in \mathfrak{a}^{*}$. We shall write

$$
\iota(\sigma, \nu) \in \widehat{G}_{r}
$$

for the equivalence class of the irreducible unitary principal series representation

$$
\pi_{\sigma, \nu}=\operatorname{Ind}_{P}^{G} \sigma \otimes e^{i \nu}
$$

of the group $G$.

Now for $f \in C_{c}^{\infty}(G)$, we define

$$
\pi_{\sigma, \nu}(f) \phi=\int_{G} f(g) \pi_{\sigma, \nu}(g) \phi d g
$$

for $\phi \in L^{2}(K)^{\sigma}$. The formula defines a $C^{*}$-algebra representation

$$
\pi_{\sigma, \nu}: C_{r}^{*}(G) \longrightarrow \mathfrak{B}\left(L^{2}(K)^{\sigma}\right) .
$$

Theorem 3.1.7 (See for example [CCH16, Cor. 4.12]). Let $G$ be a connected complex reductive group and let $\sigma \in \widehat{M}$. There is a $C^{*}$-algebra morphism

$$
\pi_{\sigma}: C_{r}^{*}(G) \longrightarrow C_{0}\left(\mathfrak{a}^{*}, \mathfrak{K}\left(L^{2}(K)^{\sigma}\right)\right)
$$

such that $\pi_{\sigma}(f)(\nu)=\pi_{\sigma, \nu}(f)$ for every $\nu \in \mathfrak{a}^{*}$.

Now let $\sigma \in \widehat{M}$, and denote by $W_{\sigma}$ the isotropy group of $\sigma$ in the Weyl group $W=N_{K}(M) / M$. Associate to $\sigma$ its infinitesimal form

$$
\sigma: \mathfrak{m} \longrightarrow i \mathbb{R}
$$

and then extend this to a linear functional

$$
\sigma: \mathfrak{k} \longrightarrow i \mathbb{R}
$$

by writing $\mathfrak{k}=\mathfrak{m} \oplus \mathfrak{m}^{\perp}$ using any $K$-invariant inner product, and defining $\sigma$ to be zero on $\mathfrak{m}^{\perp}$. We may then identify $W_{\sigma}$ with the Weyl group of the isotropy group $K_{\sigma}$ for (3.1.8). It follows, in particular, that $W_{\sigma}$ is itself a Weyl group. Moreover, since $G$ is a complex group, we have that

$$
\mathfrak{s}=i \cdot \mathfrak{k} \text { and } \mathfrak{a}=i \cdot \mathfrak{m} .
$$


So we can divide $\mathfrak{a}^{*}$ into Weyl chambers for the action of $W_{\sigma}$, and choose one positive Weyl chamber

$$
\mathfrak{a}_{+, \sigma}^{*} \subseteq \mathfrak{a}^{*},
$$

which is a fundamental domain for the action of $W_{\sigma}$ on $\mathfrak{a}^{*}$.

The morphisms $\pi_{\sigma}$ in Theorem 3.1.7 determine morphisms

$$
\pi_{\sigma}: C_{r}^{*}(G) \longrightarrow C_{0}\left(\mathfrak{a}_{+, \sigma}^{*}, \mathfrak{K}\left(L^{2}(K)^{\sigma}\right)\right)
$$

by restriction to the positive Weyl chamber. These assemble into a $C^{*}$-algebra isomorphism, as follows:

Theorem 3.1.10. Let $G$ be a complex reductive group. The representations of $G$ in the unitary principal series induce a $C^{*}$-algebra isomorphism

$$
\oplus_{\sigma \in \widehat{M}_{+}} \pi_{\sigma}: C_{r}^{*}(G) \stackrel{\cong}{\longrightarrow} \bigoplus_{\sigma \in \widehat{M}_{+}} C_{0}\left(\mathfrak{a}_{+, \sigma}^{*}, \mathfrak{K}\left(L^{2}(K)^{\sigma}\right)\right),
$$

where $\widehat{M}_{+} \subseteq \widehat{M}$ is a positive Weyl chamber for the action of $W$.

Proof. The general considerations in $\mathrm{CCH} 16$ show that there is an isomorphism

$$
\oplus_{\sigma \in \widehat{M}_{+}} \pi_{\sigma}: C_{r}^{*}(G) \stackrel{\cong}{\longrightarrow} \bigoplus_{\sigma \in \widehat{M}_{+}} C_{0}\left(\mathfrak{a}^{*}, \mathfrak{K}\left(L^{2}(K)^{\sigma}\right)\right)^{W_{\sigma}},
$$

where $W_{\sigma}$ acts by intertwining automorphisms on $C_{0}\left(\mathfrak{a}^{*}, \mathfrak{K}\left(L^{2}(K)^{\sigma}\right)\right)$. The required result now follows from the fact that $\mathfrak{a}_{+, \sigma}^{*}$ is a fundamental domain for the action of $W_{\sigma}$ on $\mathfrak{a}^{*}$.

\subsection{The motion group. The Cartan motion group}

$$
G_{0}=K \ltimes \mathfrak{g} / \mathfrak{k} \cong K \ltimes \mathfrak{s}
$$

is amenable, so its tempered dual is equal to the full unitary dual, and the canonical morphism $C^{*}\left(G_{0}\right) \rightarrow C_{r}^{*}\left(G_{0}\right)$ from the full to the reduced group $C^{*}$-algebra is an isomorphism. We shall use $C_{r}^{*}\left(G_{0}\right)$ rather than $C^{*}\left(G_{0}\right)$ in what follows simply to be consistent with our usage for the complex reductive group $G$, which is not amenable unless it is abelian.

Let $\nu \in \mathfrak{s}^{*}$. The function $\exp (i \nu)$ is a unitary character on the additive group $\mathfrak{s}$, and so we may form the unitarily induced representation

$$
\pi_{\nu}=\operatorname{Ind}_{\mathfrak{s}}^{K \ltimes \mathfrak{s}} \exp (i \nu)
$$

of the motion group $G_{0}$. By definition, its Hilbert space is the completion of the space of smooth functions $\psi: G_{0} \rightarrow \mathbb{C}$ such that

$$
\psi(k, X)=\psi(k) \exp (-i \nu(X)) \quad\left(\forall(k, X) \in G_{0}\right)
$$

in the norm induced from the inner product

$$
\langle\phi, \psi\rangle=\int_{K} \overline{\phi(k, 0)} \psi(k, 0) d k .
$$

The action of $G_{0}$ is by left translation.

Of course the Hilbert space identifies with $L^{2}(K)$. Under this identification the subgroup $K \subseteq G_{0}$ acts by left translation, whereas an element $X \in \mathfrak{s}$ acts by pointwise multiplication by the function $k \mapsto \exp \left(i \nu\left(k^{-1} \cdot X\right)\right)$. 
The unitary representation $\pi_{\nu}$ of $G_{0}$ integrates to a $C^{*}$-algebra representation

$$
\pi_{\nu}: C_{r}^{*}\left(G_{0}\right) \longrightarrow \mathfrak{K}\left(L^{2}(K)\right),
$$

and the Riemann-Lebesgue lemma for the ordinary Fourier transform implies the following result:

Lemma 3.2.1. There is a morphism $C^{*}$-algebras

$$
\pi: C_{r}^{*}\left(G_{0}\right) \longrightarrow C_{0}\left(\mathfrak{s}^{*}, \mathfrak{K}\left(L^{2}(K)\right)\right)
$$

such that

$$
\pi(f)(\nu)=\pi_{\nu}(f) \quad\left(\forall \nu \in \mathfrak{s}^{*}\right) .
$$

Now the group $K$ acts on $\mathfrak{s}^{*}$ by the coadjoint representation and on $L^{2}(K)$ by right translation, and these actions combine to give an action of $K$ on the $C^{*}$-algebra $C_{0}\left(\mathfrak{s}^{*}, \mathfrak{K}\left(L^{2}(K)\right)\right)$ by $C^{*}$-algebra automorphisms. The following result describes the $C^{*}$-algebra $C_{r}^{*}\left(G_{0}\right)$ up to isomorphism:

Theorem 3.2.2 (See for example Hig08, Thm 3.2]). The morphism $\pi$ in Lemma 3.2 .1 induces an isomorphism

$$
C_{r}^{*}\left(G_{0}\right) \stackrel{\cong}{\longrightarrow} C_{0}\left(\mathfrak{s}^{*}, \mathfrak{K}\left(L^{2}(K)\right)\right)^{K} .
$$

The representations $\pi_{\nu}$ are not irreducible, but using the theorem it is not difficult to obtain the following description of the irreducible unitary representations of $G_{0}$, which is a simple case of a general result due to Mackey (see Mac49. or [Mac76]). See also Hig08, Sec. 3.1].

Theorem 3.2.3. Let $\nu \in \mathfrak{s}^{*}$ and let $K_{\nu}$ be the subgroup of $K$ that fixes $\nu$ under the coadjoint action. If $\tau$ is an irreducible unitary representation of $K_{\nu}$, then the representation

$$
\operatorname{Ind}_{K \nu \ltimes \mathfrak{s}}^{K \ltimes \mathfrak{s}} \tau \otimes e^{i \nu}
$$

is irreducible. Every irreducible unitary representation of $G_{0}$ is equivalent to one that is obtained in this way, and two representations

$$
\operatorname{Ind}_{K_{\nu^{\prime} \ltimes \mathfrak{s}}^{K \ltimes \mathfrak{s}}}^{K} \tau^{\prime} \otimes e^{i \nu^{\prime}} \quad \text { and } \quad \operatorname{Ind}_{K_{\nu^{\prime \prime}} \ltimes \mathfrak{s}}^{K \ltimes \mathfrak{s}} \tau^{\prime \prime} \otimes e^{i \nu^{\prime \prime}}
$$

are unitarily equivalent if and only if the data $\left(\tau^{\prime}, \nu^{\prime}\right)$ and $\left(\tau^{\prime \prime}, \nu^{\prime \prime}\right)$ are conjugate by an element of $K$.

Now let $\mathfrak{a} \subseteq \mathfrak{s}$ and $M \subseteq K$ be as in the previous subsection. Because $G$ is a complex group, we can form $i \cdot \mathfrak{k} \subseteq \mathfrak{g}$, and in fact

$$
\mathfrak{s}=i \cdot \mathfrak{k},
$$

from which it follows that $\mathfrak{a}=i \cdot \mathfrak{m}$, where $\mathfrak{m}$ is the Lie algebra of $M$. It follows from standard facts about compact groups that every element of $\mathfrak{s}$ is conjugate by an element of $K$ to an element of $\mathfrak{a}$, and that two elements in $\mathfrak{a}$ are conjugate to one another by an element of $K$ if and only if they are conjugate by an element of the Weyl group $W=N_{K}(M) / M$.

We can write

$$
\mathfrak{s}=\mathfrak{a} \oplus \mathfrak{a}^{\perp}
$$

using a $K$-invariant inner product on $\mathfrak{s}$ (the orthogonal complement does not depend of the choice of inner product), and so regard $\mathfrak{a}^{*}$ as a subspace of $\mathfrak{s}^{*}$. We see from 
Theorem 3.2.3, therefore, that every irreducible representation of $G_{0}$ is equivalent to one of the form

$$
\operatorname{Ind}_{K \nu \ltimes \mathfrak{s}}^{K \ltimes \mathfrak{s}} \tau \otimes e^{i \nu}
$$

for some $\nu \in \mathfrak{a}^{*}$ and some $\tau \in \widehat{K}_{\nu}$

Finally, $K_{\nu}$ is a connected compact Lie group for every $\nu \in \mathfrak{a}^{*}$, and $M \subseteq K_{\nu}$ is a maximal torus. So the irreducible representations of $K_{\nu}$ are parametrized by their highest weights, which are orbits in $\widehat{M}$ of the Weyl group

$$
W_{\nu}=N_{K_{\nu}}(M) / M,
$$

which is also the isotropy group of $\nu \in \mathfrak{a}^{*}$ for the action of $W$ on $\mathfrak{a}^{*}$. So the irreducible unitary representations of $G_{0}$ are parametrized by elements of the set

$$
\left(\bigsqcup_{\nu \in \mathfrak{a}^{*}} \widehat{M} / W_{\nu} \times\{\nu\}\right) / W=\left(\widehat{M} \times \mathfrak{a}^{*}\right) / W .
$$

For future use we shall introduce the following notation for irreducible representation attached to the parameter $(\sigma, \nu)$ :

Definition 3.2.4. Let $\sigma \in \widehat{M}$ and let $\nu \in \mathfrak{a}^{*}$. Let $\tau_{\sigma} \in \widehat{K}_{\nu}$ be the irreducible representation with highest weight $[\sigma] \in \widehat{M} / W_{\nu}$. We shall write

$$
\pi(\sigma, \nu) \in \widehat{G}_{0}
$$

for the equivalence class of the irreducible unitary representation

$$
\operatorname{Ind}_{K}^{K \ltimes \mathfrak{s}} \tau_{\nu} \otimes e^{i \nu}
$$

of the motion group $G_{0}$.

\section{SCALing AUtomorphisms}

In this section we shall construct a one-parameter group of automorphisms

$$
\alpha_{t}: C_{r}^{*}(G) \longrightarrow C_{r}^{*}(G) .
$$

The automorphisms will be parametrized by the multiplicative group of positive real numbers, rather than the usual additive group of real numbers, and so the group law is $\alpha_{t_{1}} \circ \alpha_{t_{2}}=\alpha_{t_{1} t_{2}}$.

4.1. Definition of the scaling automorphisms. Given the structure theory for $C_{r}^{*}(G)$ that was presented in the previous section, the construction is extremely simple. Let $\sigma \in \widehat{M}_{+}$. Define, for $t>0$, an automorphism

$$
\alpha_{\sigma, t}: C_{0}\left(\mathfrak{a}_{+, \sigma}^{*}, \mathfrak{K}\left(L^{2}(K)^{\sigma}\right)\right) \longrightarrow C_{0}\left(\mathfrak{a}_{+, \sigma}^{*}, \mathfrak{K}\left(L^{2}(K)^{\sigma}\right)\right)
$$

by

$$
\alpha_{\sigma, t}(f)(\nu)=f\left(t^{-1} \nu\right) .
$$

The individual one-parameter groups $\alpha_{\sigma, t}$ may be combined by direct sum into a one-parameter group of automorphisms

$$
\oplus_{\sigma} \alpha_{\sigma, t}: \bigoplus_{\sigma \in \widehat{M}_{+}} C_{0}\left(\mathfrak{a}_{+, \sigma}^{*}, \mathfrak{K}\left(L^{2}(K)^{\sigma}\right)\right) \longrightarrow \bigoplus_{\sigma \in \widehat{M}_{+}} C_{0}\left(\mathfrak{a}_{+, \sigma}^{*}, \mathfrak{K}\left(L^{2}(K)^{\sigma}\right)\right),
$$


and then we define automorphisms $\alpha_{t}$ of $C_{r}^{*}(G)$ by means of the commuting diagram $(4.1 .2)$

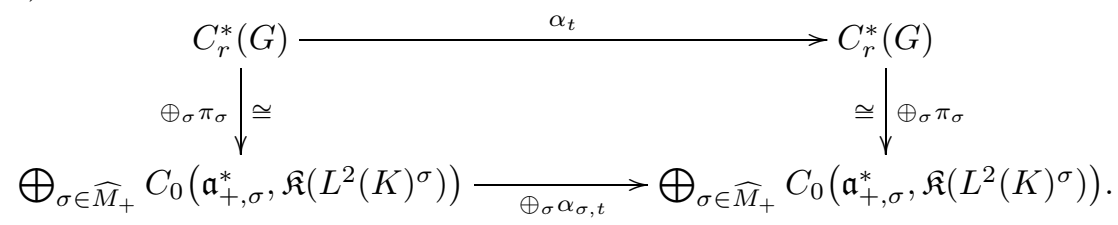

4.2. Scaling automorphisms for negative t. As we shall soon see, the key property of the rescaling automorphism $\alpha_{t}$, which is immediate from its definition, is that if $\sigma \in \widehat{M}_{+}$and if $\nu \in \mathfrak{a}_{\sigma,+}^{*}$, then

$$
\pi_{\sigma, \nu}\left(\alpha_{t}(f)\right)=\pi_{\sigma, t^{-1} \nu}(f)
$$

for all $f \in C_{r}^{*}(G)$. We shall want to extend this to negative $t$, and to this end we define automorphisms

for $t<0$ as follows.

$$
\alpha_{t}: C_{r}^{*}(G) \longrightarrow C_{r}^{*}(G)
$$

First we define $\mathfrak{a}_{-, \sigma}^{*}$ to be the negative of the Weyl chamber $\mathfrak{a}_{+, \sigma}^{*}$. This is simply another Weyl chamber for $W_{\sigma}$, and so all the constructions that we made in Section 3 using $\mathfrak{a}_{+, \sigma}^{*}$ can be repeated for $\mathfrak{a}_{-, \sigma}^{*}$. In particular there is an isomorphism of $C^{*}$-algebras

$$
\oplus_{\sigma \in \widehat{M}_{+}} \pi_{\sigma}: C_{r}^{*}(G) \stackrel{\cong}{\longrightarrow} \bigoplus_{\sigma \in \widehat{M}_{+}} C_{0}\left(\mathfrak{a}_{-, \sigma}^{*}, \mathfrak{K}\left(L^{2}(K)^{\sigma}\right)\right) .
$$

We now define $\alpha_{t}: C_{r}^{*}(G) \rightarrow C_{r}^{*}(G)$ for $t<0$ by means of the commuting diagram

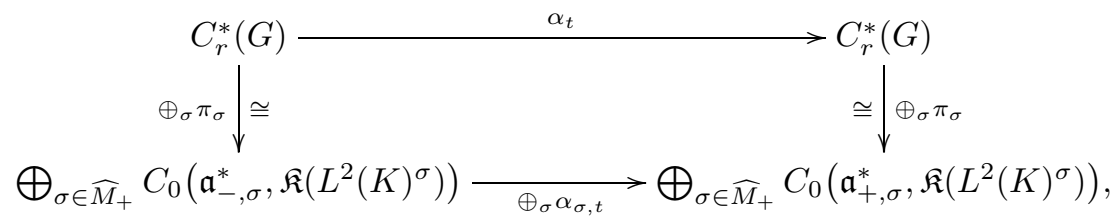

where $\alpha_{\sigma, t}(h)(\nu)=h\left(t^{-1} \nu\right)$. The key property (4.1.1) now holds for all $t \neq 0$, for all $\nu \in \mathfrak{a}_{+, \sigma}^{*}$ and all $f \in C_{r}^{*}(G)$.

\section{Limit FORMULA AND EMBEDDING}

5.1. Limit formula. The main result of this section links the scaling automorphisms

$$
\alpha_{t}: C_{r}^{*}(G) \rightarrow C_{r}^{*}(G)
$$

from (4.1.2) with the regular representations

$$
\lambda_{t}: C_{r}^{*}\left(G_{t}\right) \rightarrow C_{r}^{*}(G)
$$

from (2.2.3) as follows:

Theorem 5.1.1. If $\left\{f_{t}\right\}$ is any continuous section of the continuous field $\left\{C_{r}^{*}\left(G_{t}\right)\right\}$, then the limit

$$
\lim _{t \rightarrow 0} \alpha_{t}\left(\lambda_{t}\left(f_{t}\right)\right)
$$

exists in $C_{r}^{*}(G)$.

Remark 5.1.2. Of course we exclude the value $t=0$ in forming the limit. 
We shall prove the theorem by carrying out an explicit computation with a suitable collection of continuous sections. To this end, recall that a collection $\mathcal{F}$ of continuous sections of the continuous field of $C^{*}$-algebras $\left\{C_{r}^{*}\left(G_{t}\right)\right\}$ is called a generating family if for every continuous section $s$, every $\varepsilon>0$ and every $t_{0} \in \mathbb{R}$ there is some element $f \in \mathcal{F}$ and a neighborhood $U$ of $t_{0} \in \mathbb{R}$ such that

$$
t \in U \quad \Rightarrow \quad\|f(t)-s(t)\|<\varepsilon
$$

(compare Dix77, 10.2.3]). The following is an immediate consequence of the fact that $C^{*}$-algebra isomorphisms are isometric:

Lemma 5.1.3. If the limit in Theorem 5.1.1 exists for a generating family of continuous sections of $\left\{C_{r}^{*}\left(G_{t}\right)\right\}$, then it exists for all continuous sections of $\left\{C_{r}^{*}\left(G_{t}\right)\right\}$.

Recall that if $K$ acts continuously on a complex vector space $W$, then a vector $w \in W$ is said to be $K$-finite if the linear span of the orbit of $w$ under the action of $K$ is finite-dimensional and the action on this finite-dimensional space is continuous, or equivalently if $w$ lies in the image under the natural map

$$
\bigoplus_{\tau \in \widehat{K}} V_{\tau} \otimes_{\mathbb{C}} \operatorname{Hom}_{K}\left(V_{\tau}, W\right) \longrightarrow W
$$

of the span of finitely many summands $V_{\tau} \otimes_{\mathbb{C}} \operatorname{Hom}_{K}\left(V_{\tau}, W\right)$ (here $V_{\tau}$ is the representation space for a representative of $\tau \in \widehat{K})$. We shall call the minimal set of $\tau \in \widehat{K}$ here the $K$-isotypical support of $w \in W$.

Lemma 5.1.4. There exists a generating family of continuous sections for the continuous field $\left\{C_{r}^{*}\left(G_{t}\right)\right\}$ consisting of smooth and compactly supported functions on $\mathbb{N}_{G} K$ that are $K$-finite for both the left and right translation actions of $K$ on $\mathbb{N}_{G} K$.

Proof. In the following argument we shall use right and left convolutions of elements $\phi \in C^{\infty}(K)$, or even $\phi \in L^{2}(K)$, with elements $f \in C_{c}^{\infty}\left(\mathbb{N}_{K} G\right)$, defined by

$$
(f * \phi)(g)=\int_{K} f\left(g k^{-1}\right) \phi(k) d k
$$

and

$$
(\phi * f)(g)=\int_{K} \phi(k) f\left(k^{-1} g\right) d k .
$$

Both convolutions are smooth and compactly supported functions on $\mathbb{N}_{G} K$. The formulas define $C^{*}$-algebra morphisms from $C_{r}^{*}(K)$ into the multiplier algebra of the $C^{*}$-algebra of continuous sections of $\left\{C_{r}^{*}\left(G_{t}\right)\right\}$ that vanish at infinity.

Given a smooth and compactly supported complex function $f$ on $\mathbb{N}_{G} K$ and $\varepsilon>0$, we can find smooth functions $\phi$ and $\psi$ on $K$ such that

$$
\|f-\phi * f * \psi\|<\varepsilon
$$

where the norm is that of the $C^{*}$-algebra of continuous sections of $\left\{C_{r}^{*}\left(G_{t}\right)\right\}$ that vanish at infinity. Now recall the Peter-Weyl isomorphism

$$
C_{r}^{*}(K) \stackrel{\cong}{\longrightarrow} \bigoplus_{\tau \in \widehat{K}} \operatorname{End}\left(V_{\tau}\right) .
$$


We can approximate $\phi$ and $\psi$, viewed as elements of $C_{r}^{*}(K)$, by elements $\phi_{1}$ and $\psi_{1}$ of the $C^{*}$-algebra that map into the algebraic direct sum above, in such a way that

$$
\left\|f-\phi_{1} * f * \psi_{1}\right\|<\varepsilon .
$$

The elements $\phi_{1}, \psi_{1} \in C_{r}^{*}(K)$ are automatically smooth functions on $K$, and the function $\phi_{1} * f * \psi_{1}$ on $\mathbb{N}_{G} K$ is smooth, compactly supported and left and right $K$-finite. The collection of all elements of this form, for all $f$ and all $\varepsilon>0$, is a generating family, as required.

In the proofs of the following two lemmas we shall use the fact that if $f \in C_{c}^{\infty}(G)$ and $\phi \in C^{\infty}(K)$, then

$$
\pi_{\sigma, \nu}(f * \phi)=\pi_{\sigma, \nu}(f) \pi_{\sigma}(\phi) \quad \text { and } \quad \pi_{\sigma, \nu}(\phi * f)=\pi_{\sigma}(\phi) \pi_{\sigma, \nu}(f),
$$

where $\pi_{\sigma}$ denotes the restriction of the representation $\pi_{\sigma, \nu}$ to $K$. The restriction is independent of $\nu$; it is simply the restriction of the left-regular representation of $K$ to $L^{2}(K)^{\sigma} \subseteq L^{2}(K)$.

Lemma 5.1.5. Let $\left\{f_{t}\right\}$ be a right $K$-finite continuous section of $\left\{C_{r}^{*}\left(G_{t}\right)\right\}$. If for every $\sigma \in \widehat{M}_{+}$the limit

$$
\lim _{t \rightarrow 0} \pi_{\sigma}\left(\alpha_{t}\left(\lambda_{t}\left(f_{t}\right)\right)\right)
$$

exists in $C_{0}\left(\mathfrak{a}_{+, \sigma}^{*}, \mathfrak{K}\left(L^{2}(K)^{\sigma}\right)\right)$, then the limit

$$
\lim _{t \rightarrow 0} \alpha_{t}\left(\lambda_{t}\left(f_{t}\right)\right)
$$

exists in $C_{r}^{*}(G)$.

Proof. By the Peter-Weyl theorem, the Hilbert space $L^{2}(K)^{\sigma}$ has a $K$-isotypical decomposition

$$
L^{2}(K)^{\sigma} \cong \bigoplus_{\tau \in \widehat{K}} V_{\tau} \otimes \operatorname{Hom}_{K}\left(V_{\tau}, \mathbb{C}_{\sigma}\right)
$$

It follows that an irreducible representation $\tau \in \widehat{K}$, is included in the $K$-isotypical decomposition of $L^{2}(K)^{\sigma}$ if and only if $\sigma$ is a weight of $\tau$, and therefore $\tau$ is included in only finitely many of the spaces $L^{2}(K)^{\sigma}$, as $\sigma$ ranges over $\widehat{M}$. So if $f$ is right $K$-finite, then the element

$$
\pi_{\sigma}\left(\lambda_{t}\left(f_{t}\right)\right) \in C_{0}\left(\mathfrak{a}_{+, \sigma}^{*}, \mathfrak{K}\left(L^{2}(K)^{\sigma}\right)\right.
$$

is nonzero for only a finite set of $\sigma \in \widehat{M}$ that is independent of $t$. Therefore under the hypotheses of the lemma the limit

$$
\lim _{t \rightarrow 0} \bigoplus_{\sigma} \pi_{\sigma}\left(\alpha_{t}\left(\lambda_{t}\left(f_{t}\right)\right)\right)=\bigoplus_{\sigma} \lim _{t \rightarrow 0} \pi_{\sigma}\left(\alpha_{t}\left(\lambda_{t}\left(f_{t}\right)\right)\right)
$$

exists: we can commute the limit and the direct sum because only finitely many summands are nonzero. The lemma follows from the fact that $\oplus_{\sigma} \pi_{\sigma}$ is isometric.

Lemma 5.1.6. Let $\left\{f_{t}\right\}$ be a left and right $K$-finite continuous section of $\left\{C_{r}^{*}\left(G_{t}\right)\right\}$ and let $\sigma \in \widehat{M}$. If the limit

$$
\lim _{t \rightarrow 0}\left\langle\phi, \pi_{\sigma, \nu}\left(\alpha_{t}\left(\lambda_{t}\left(f_{t}\right)\right)\right) \psi\right\rangle
$$


exists for every $\phi, \psi \in L^{2}(K)^{\sigma}$, uniformly in $\nu \in \mathfrak{a}^{*}$, then the limit

$$
\lim _{t \rightarrow 0} \pi_{\sigma}\left(\alpha_{t}\left(\lambda_{t}\left(f_{t}\right)\right)\right)
$$

exists in $C_{0}\left(\mathfrak{a}_{+, \sigma}^{*}, \mathfrak{K}\left(L^{2}(K)^{\sigma}\right)\right)$.

Proof. Let $S \subseteq \widehat{K}$ be the union of the $K$-isotypical supports of $\left\{f_{t}\right\}$ for the left and right translation actions, and let

$$
\left.L^{2}(K)_{S}^{\sigma}=\operatorname{Image}\left(\bigoplus_{\tau \in S} V_{\tau} \otimes \operatorname{Hom}_{K}\left(V_{\tau}, L^{2}(K)^{\sigma}\right) \longrightarrow L^{2}(K)^{\sigma}\right)\right) .
$$

This is a finite-dimensional subspace of $L^{2}(K)^{\sigma}$, and the operators $\pi_{\sigma, \nu}\left(f_{t}\right)$ vanish on its orthogonal complement for all $\nu$ and all $t$. If for any given $\nu \in \mathfrak{a}^{*}$ the limits

$$
\lim _{t \rightarrow 0}\left\langle\phi, \pi_{\sigma, \nu}\left(\alpha_{t}\left(\lambda_{t}\left(f_{t}\right)\right)\right) \psi\right\rangle
$$

exist for all $\phi, \psi \in L^{2}(K)_{S}^{\sigma}$, then the limit

$$
\lim _{t \rightarrow 0} \pi_{\sigma, \nu}\left(\alpha_{t}\left(\lambda_{t}\left(f_{t}\right)\right)\right)
$$

exists in $\mathfrak{K}\left(L^{2}(K)^{\sigma}\right)$. If the limits (5.1.7) exist uniformly in $\nu$ as $\phi$ and $\psi$ range over an orthonormal basis for $\phi, \psi \in L^{2}(K)_{S}^{\sigma}$, then the limit

$$
\lim _{t \rightarrow 0} \pi_{\sigma}\left(\alpha_{t}\left(\lambda_{t}\left(f_{t}\right)\right)\right)
$$

exists in $C_{0}\left(\mathfrak{a}_{+, \sigma}^{*}, \mathfrak{K}\left(L^{2}(K)^{\sigma}\right)\right)$, as required.

We shall use the following explicit formula for the Haar integral on $G_{t}$ (recall here that for $t \neq 0$ the Haar measure on $G_{t}$ is $\left.|t|^{-d} d g\right)$.

Lemma 5.1.8 (See [Kna02, Prop 8.43]). If $G=K A N$ is an Iwasawa decomposition, then the Haar measures on $K, \mathfrak{a}$, and $\mathfrak{n}$ can be normalized so that

$$
\begin{aligned}
\int_{G_{t}} f\left(g_{t}\right) d g_{t} & =\int_{K} \int_{\mathfrak{a}} \int_{\mathfrak{n}} f(k \exp (X) \exp (Y)) e^{2 \rho(X)}|t|^{-d} d k d X d Y \\
& =\int_{K} \int_{\mathfrak{a}} \int_{\mathfrak{n}} f(k \exp (t X) \exp (t Y)) e^{2 \rho(t X)} d k d X d Y
\end{aligned}
$$

Lemma 5.1.9. Let $\sigma \in \widehat{M}_{+}$, let $\nu \in \mathfrak{a}_{+, \sigma}^{*}$ and let $t \neq 0$. If $f_{t} \in C_{r}^{*}\left(G_{t}\right)$ is represented by a smooth and compactly supported function on $G_{t}$, and if $\phi, \psi \in C^{\infty}(K)^{\sigma}$, then

$$
\begin{aligned}
\left\langle\phi, \pi_{\sigma, \nu}\left(\alpha_{t}\left(\lambda_{t}\left(f_{t}\right)\right)\right) \psi\right\rangle & \\
& =\int_{\mathfrak{a}} \int_{\mathfrak{n}}\left(\phi^{*} * f_{t} * \psi\right)(\exp (-t Y) \exp (-t X)) e^{i \nu(X)} e^{t \rho(X)} d X d Y,
\end{aligned}
$$

where $\phi^{*}(k)=\overline{\phi\left(k^{-1}\right)}$.

Proof. It follows from the definitions of the scaling automorphism $\alpha_{t}$ and the morphism $\pi_{\sigma}$ that

$$
\left\langle\phi, \pi_{\sigma, \nu}\left(\alpha_{t}\left(\lambda_{t}\left(f_{t}\right)\right)\right) \psi\right\rangle=\int_{G} f_{t}(g)\left\langle\phi, \pi_{\sigma, t^{-1} \nu}(g) \psi\right\rangle|t|^{-d} d g .
$$


If we insert into this formula the definition of the $L^{2}$-inner product on the induced representation space, then we obtain

$$
\begin{aligned}
\int_{G} f_{t}(g)\left\langle\phi, \pi_{\sigma, t^{-1} \nu}(g) \psi\right\rangle \mid & ||^{-d} d g \\
& =\int_{G} f_{t}(g)\left(\int_{K} \phi^{*}\left(k^{-1}\right)\left(\pi_{\sigma, t^{-1} \nu}(g) \psi\right)(k) d k\right)|t|^{-d} d g,
\end{aligned}
$$

and rearranging, and making the substitution $g:=k \gamma^{-1}$, we get

$$
\begin{aligned}
\int_{G} f_{t}(g)\langle\phi, & \left.\pi_{\sigma, t^{-1} \nu}(g) \psi\right\rangle|t|^{-d} d g \\
& =\int_{K} \int_{G} \phi^{*}\left(k^{-1}\right) f_{t}\left(k \gamma^{-1}\right)\left(\pi_{\sigma, t^{-1} \nu}\left(k \gamma^{-1}\right) \psi\right)(k) d k|t|^{-d} d \gamma
\end{aligned}
$$

Now, according to the definition (3.1.3) of the principal series representations,

$$
\left(\pi_{\sigma, t^{-1} \nu}\left(k \gamma^{-1}\right) \psi\right)(k)=e^{-\left(\rho+i t^{-1} \nu\right) H(\gamma)} \psi(\kappa(\gamma)) .
$$

Inserting this into the right-hand side of (5.1.10) we obtain

$$
\int_{K} \int_{G} \phi^{*}\left(k^{-1}\right) f_{t}\left(k \gamma^{-1}\right) e^{\left(-\rho+i t^{-1} \nu\right) H(\gamma)} \psi(\kappa(\gamma))|t|^{-d} d \gamma d k .
$$

If we use the formula for the Haar measure on $G$ given in Lemma [5.1.8, then we obtain from (5.1.11) the integral

$$
\begin{aligned}
& \int_{K} \int_{\mathfrak{a}} \int_{\mathfrak{n}} \int_{K} \phi^{*}\left(k_{1}^{-1}\right) f_{t}\left(k_{1} \exp (\right.\left.-t Y) \exp (-t X) k_{2}^{-1}\right) \\
& \times \phi\left(k_{2}\right) e^{\left(-\rho+i t^{-1} \nu\right)(t X)} e^{2 \rho(t X)} d k_{1} d X d Y d k_{2} .
\end{aligned}
$$

This is

$$
\int_{\mathfrak{a}} \int_{\mathfrak{n}}\left(\phi^{*} * f_{t} * \psi\right)(\exp (-t Y) \exp (-t X)) e^{i \nu(X)} e^{\rho(t X)} d X d Y
$$

as required.

Lemma 5.1.12. Let $\sigma \in \widehat{M}_{+}$and let $\nu \in \mathfrak{a}_{+, \sigma}^{*}$. For any smooth and compactly supported function $f$ on $\mathbb{N}_{G} K$, and any $\phi, \psi \in L^{2}(K)^{\sigma}$, we have

$$
\lim _{t \rightarrow 0}\left\langle\phi, \pi_{\sigma, \nu}\left(\alpha_{t}\left(\lambda_{t}\left(f_{t}\right)\right)\right) \psi\right\rangle=\int_{\mathfrak{a}} \int_{\mathfrak{n}}\left(\phi^{*} * f_{0} * \psi\right)(e, X+Y) e^{i \nu(X)} d X d Y .
$$

The convergence is uniform in $\nu \in \mathfrak{a}_{+, \sigma}^{*}$.

Proof. This follows immediately from Lemma 5.1 .9 above and Lemma 2.3.2

Proof of Theorem 5.1.1. According to Lemma 5.1.3, we only need verify that the limit in the statement of the theorem exists for a generating family of continuous sections, and we shall use Lemma 5.1.4 to work with the generating family of continuous sections $\left\{f_{t}\right\}$ associated to the smooth, compactly supported, left and right $K$-finite functions on $\mathbb{N}_{G} K$. Lemma 5.1 .12 shows that for every $\sigma \in \widehat{M}_{+}$and every $\nu \in \mathfrak{a}_{\sigma,+}^{*}$ the individual matrix coefficients of $\pi_{\sigma, \nu}\left(\alpha_{t}\left(\lambda_{t}(f)\right)\right)$ converge to limits as $t \rightarrow 0$, uniformly in $\nu$. Lemmas 5.1.5 and 5.1.6 complete the proof. 
5.2. Construction of an embedding morphism. Let $f \in C_{r}^{*}\left(G_{0}\right)$. Extend $f$ in any way to a continuous section $\left\{f_{t}\right\}$ of $\left\{C_{r}^{*}\left(G_{t}\right)\right\}$ and then form the limit

$$
\alpha(f)=\lim _{t \rightarrow 0} \alpha_{t}\left(\lambda_{t}\left(f_{t}\right)\right)
$$

in $C_{r}^{*}(G)$.

Theorem 5.2.2. The formula (5.2.1) defines an embedding of $C^{*}$-algebras

$$
\alpha: C_{r}^{*}\left(G_{0}\right) \longrightarrow C_{r}^{*}(G) .
$$

Proof. Since both $\lambda_{t}$ and $\alpha_{t}$ are isometric,

$$
\left\|\lim _{t \rightarrow 0} \alpha_{t}\left(\lambda_{t}\left(f_{t}\right)\right)\right\|=\lim _{t \rightarrow 0}\left\|f_{t}\right\|=\left\|f_{0}\right\| .
$$

Moreover if $\left\{f_{t}^{\prime}\right\}$ is a second extension of $f$ to a continuous section, then

$$
\left\|\lim _{t \rightarrow 0} \alpha_{t}\left(\lambda_{t}\left(f_{t}\right)\right)-\lim _{t \rightarrow 0} \alpha_{t}\left(\lambda_{t}\left(f_{t}^{\prime}\right)\right)\right\|=\left\|\lim _{t \rightarrow 0} \alpha_{t}\left(\lambda_{t}\left(f_{t}-f_{t}^{\prime}\right)\right)\right\|=0 .
$$

So the limit is independent of the extension, and it defines an isometric $*$-homomorphism, as required.

5.3. Mapping cone fields. We begin with a very elementary construction:

Definition 5.3.1. Let $\beta: B \rightarrow A$ be an embedding of a $C^{*}$-algebra $B$ into a $C^{*}$-algebra $A$. The mapping cone field of $C^{*}$-algebras over $\mathbb{R}$ associated to $\beta$ has fibers

$$
\operatorname{Cone}(\beta)_{t}= \begin{cases}A & t \neq 0 \\ B & t=0\end{cases}
$$

Its continuous sections are all those set-theoretic sections $\left\{f_{t}\right\}$ for which the function

$$
t \mapsto \begin{cases}f_{t} & t \neq 0 \\ \beta\left(f_{0}\right) & t=0\end{cases}
$$

from $\mathbb{R}$ to $A$ is norm-continuous.

We shall apply this construction to the embedding from Theorem 5.2 .2

Theorem 5.3.2. The fiber isomorphisms

$$
\left\{\begin{array}{cc}
\alpha_{t} \circ \lambda_{t}: C_{r}^{*}\left(G_{t}\right) \longrightarrow C_{r}^{*}(G) & t \neq 0 \\
\operatorname{id}: C_{r}^{*}\left(G_{0}\right) \longrightarrow C_{r}^{*}\left(G_{0}\right) & t=0
\end{array}\right.
$$

define an isomorphism of continuous fields from the deformation field $\left\{C_{r}^{*}\left(G_{t}\right)\right\}$ to the mapping cone field for the embedding

$$
\alpha: C_{r}^{*}\left(G_{0}\right) \longrightarrow C_{r}^{*}(G) .
$$

Proof. It suffices to show that for any continuous section $\left\{f_{t}\right\}$ of $\left\{C_{r}^{*}\left(G_{t}\right)\right\}$, the image section of the mapping cone field is continuous; see [Dix77, 10.2.4]. But the image section is $\left\{\widehat{f}_{t}\right\}$, where

$$
\widehat{f}_{t}= \begin{cases}\alpha_{t}\left(\lambda_{t}\left(f_{t}\right)\right) & t \neq 0 \\ f_{0} & t=0 .\end{cases}
$$

This is obviously a continuous section of the mapping cone field away from $t=0$, and continuity at $t=0$ is proved using Theorem 5.1.1 and the definition of $\alpha$. 


\section{Characterization of the Mackey BiJection}

The previous accounts of the Mackey bijection have all been organized around the concept of minimal $K$-type of an irreducible representation of $G$. Compare AA19. In this final section we shall give a different treatment that is organized around the embedding

$$
\alpha: C_{r}^{*}\left(G_{0}\right) \longrightarrow C_{r}^{*}(G),
$$

and hence around the family of rescaling automorphisms $\left\{\alpha_{t}\right\}$.

\subsection{The principal series as representations of the motion group.}

Lemma 6.1.1. The composition of the principal series representation $\operatorname{Ind}_{P}^{G} \sigma \otimes \nu$ of the connected complex reductive group $G$ with the morphism

$$
\alpha: C_{r}^{*}\left(G_{0}\right) \longrightarrow C_{r}^{*}(G)
$$

is the unitary representation $\operatorname{Ind}_{M \ltimes \mathfrak{s}}^{K \ltimes \mathfrak{s}} \sigma \otimes \exp (i \nu)$ of the motion group $G_{0}$.

Proof. The Hilbert space of the representation $\pi=\operatorname{Ind}_{M \ltimes \mathfrak{s}}^{K \ltimes \mathfrak{s}} \sigma \otimes \exp (i \nu)$ is the completion of the space of smooth functions $f: G_{0} \rightarrow \mathbb{C}$ such that

$$
f(g \cdot(m, X))=\sigma(m)^{-1} \exp (-i \nu(X)) f(g)
$$

in the norm associated to the inner product

$$
\left\langle f_{1}, f_{2}\right\rangle=\int_{K} \overline{f_{1}(k)} f_{2}(k) d k .
$$

The action of $G_{0}$ is by left translation. The Hilbert space identifies with $L^{2}(K)^{\sigma}$ by restriction of functions to $K$, and in this realization the action of $G_{0}$ is

$$
(\pi(k, X) \psi)\left(k_{1}\right)=\exp \left(i \nu\left(k_{1}^{-1} k \cdot X\right)\right) \psi\left(k^{-1} k_{1}\right) .
$$

The matrix coefficient associated to $\phi, \psi \in L^{2}(K)$ and $f \in C_{c}^{\infty}\left(G_{0}\right)$ is therefore

$$
\langle\phi, \pi(f) \psi\rangle=\int_{K} \int_{K} \int_{\mathfrak{s}} \overline{\phi\left(k_{1}\right)} f(k, X) \exp \left(i \nu\left(k_{1}^{-1} k \cdot X\right)\right) \psi\left(k^{-1} k_{1}\right) d k d k_{1} d X .
$$

Making the change of variables $k_{2}:=k_{1}^{-1} k$ we get

$$
\langle\phi, \pi(f) \psi\rangle=\int_{K} \int_{\mathfrak{s}} \int_{K} \overline{\phi\left(k_{1}\right)} f\left(k_{1} k_{2}, X\right) \exp \left(i \nu\left(k_{2} \cdot X\right)\right) \psi\left(k_{2}^{-1}\right) d k_{1} d X d k_{2},
$$

and then the further change of variables $Z:=k_{2} \cdot X$ gives

$$
\langle\phi, \pi(f) \psi\rangle=\int_{K} \int_{\mathfrak{s}} \int_{K} \overline{\phi\left(k_{1}\right)} f\left(k_{1} k_{2}, k_{2}^{-1} \cdot Z\right) \exp (i \nu(Z)) \psi\left(k_{2}^{-1}\right) d k_{1} d Z d k_{2} .
$$

Now let us insert into the integral above the formula

$$
\begin{aligned}
\left(\phi^{*} * f * \psi\right)(k, Z) & =\int_{K} \int_{K} \overline{\phi\left(k_{1}\right)} f\left(k_{1} \cdot(k, Z) \cdot k_{2}\right) \psi\left(k_{2}^{-1}\right) d k_{1} d k_{2} \\
& =\int_{K} \int_{K} \overline{\phi\left(k_{1}\right)} f\left(k_{1} k k_{2}, k_{2}^{-1} \cdot Z\right) \psi\left(k_{2}^{-1}\right) d k_{1} d k_{2} .
\end{aligned}
$$

We obtain

$$
\langle\phi, \pi(f) \psi\rangle=\int_{\mathfrak{s}}\left(\phi^{*} * f * \psi\right)(e, Z) \exp (i \nu(Z)) d Z .
$$

But Lemma 5.1 .12 shows that this is precisely $\left\langle\phi, \pi_{\sigma, \nu} \circ \alpha(f) \psi\right\rangle$, and the proof is complete. 
In the following lemma and in the next subsection we shall make use of the classification of irreducible representations of the compact connected group $K_{\nu}$ by highest weights. Rather than choose a dominant Weyl chamber, as is standard, it will be more convenient to associate to a given irreducible representation $\tau$ the $W_{\nu}$-orbit of all possible highest weights, for all possible choices of dominant Weyl chamber.

We shall use brackets, as in $[\sigma]$, to denote orbit of a weight $\sigma \in \widehat{M}$ under the action of the Weyl group $W_{\nu}$. The highest weight orbit $[\theta]$ of an irreducible representation $\tau$ of $K_{\nu}$ is extremal in the following sense. If $\sigma$ is any weight of $\tau$, then $[\sigma]$ lies in the convex span of $[\theta]$. Here, to form the convex hull, we view the free abelian group of all weights as a lattice in a vector space via the embedding

$$
\widehat{M} \cong \widehat{M} \otimes_{\mathbb{Z}} \mathbb{Z} \subseteq \widehat{M} \otimes_{\mathbb{Z}} \mathbb{R} .
$$

We shall write $[\sigma] \leq[\theta]$ to denote the inclusion of $[\sigma]$ in the convex hull of $[\theta]$. Of course, this partial order depends on $\nu$, but the choice of $\nu$ will be clear from the context.

Lemma 6.1.2. The composition of the principal series representation

$$
\pi_{\sigma, \nu}=\operatorname{Ind}_{P}^{G} \sigma \otimes \exp (i \nu)
$$

of the connected complex reductive group $G$ with the morphism

$$
\alpha: C_{r}^{*}\left(G_{0}\right) \longrightarrow C_{r}^{*}(G)
$$

decomposes as a direct sum

$$
\bigoplus_{[\theta] \in \widehat{M} / W_{\nu}} m(\sigma, \theta) \cdot \pi(\theta, \nu)
$$

as a representation of $G_{0}$, where the integer $m(\sigma, \theta)$ is the multiplicity with which the weight $\sigma$ occurs in the representation of $K_{\nu}$ with highest weight $\theta$.

Proof. We showed in the Lemma 6.1.1 that $\pi_{\sigma, \nu} \circ \alpha$ is the induced representation $\operatorname{Ind}_{M \ltimes \mathfrak{s}}^{K \ltimes \mathfrak{s}} \sigma \otimes \exp (i \nu)$. Let us analyze this representation by induction in stages [Mac76, Thm. 3.3 p.137]:

$$
\operatorname{Ind}_{M \ltimes \mathfrak{s}}^{K \ltimes \mathfrak{s}} \sigma \otimes \exp (i \nu) \cong \operatorname{Ind}_{K_{\nu} \ltimes \mathfrak{s}}^{K \ltimes \mathfrak{s}} \operatorname{Ind}_{M \ltimes \mathfrak{s}}^{K_{\nu} \ltimes \mathfrak{s}} \sigma \otimes \exp (i \nu) .
$$

As in the proof of Lemma 6.1.1, we can realize $\operatorname{Ind}_{M \ltimes \mathfrak{s}}^{K_{\nu} \ltimes \mathfrak{s}} \sigma \otimes \exp (i \nu)$ on the Hilbert space $L^{2}\left(K_{\nu}\right)^{\sigma}$, and in this realization an element $X \in \mathfrak{s}$ acts as multiplication by the function

$$
k \longmapsto \exp \left(i \nu\left(k^{-1} \cdot X\right)\right) \quad\left(\forall k \in K_{\nu}\right) .
$$

But if $k \in K_{\nu}$ then by definition, $\nu\left(k^{-1} \cdot X\right)=\nu(X)$. So the subgroup $\mathfrak{s}$ of $K_{\nu} \ltimes \mathfrak{s}$ acts on $L^{2}\left(K_{\nu}\right)^{\sigma}$ by the unitary character $\exp (i \nu)$. It follows that

$$
\operatorname{Ind}_{M \ltimes \mathfrak{s}}^{K_{\nu} \ltimes \mathfrak{s}} \sigma \otimes \exp (i \nu)=\bigoplus_{\tau \in \widehat{K}_{\nu}} m(\tau) \tau \otimes \exp (i \nu),
$$

where $m(\tau)$ is the multiplicity with which $\tau \in \widehat{K}_{\nu}$ occurs in $L^{2}\left(K_{\nu}\right)^{\sigma}$. By the PeterWeyl theorem (or Frobenius reciprocity) $m(\tau)$ is also the multiplicity with which the weight $\sigma$ occurs in $\tau$; compare the proof of Lemma [5.1.5. The lemma follows from this and the classification of irreducible representations of the connected group $K_{\nu}$ by their highest weights. 


\subsection{Characterization of the Mackey bijection.}

Theorem 6.2.1. There is a unique bijection

$$
\mu: \widehat{G}_{r} \longrightarrow \widehat{G}_{0}
$$

such that for every $\iota \in \widehat{G}_{r}$, the element $\mu(\iota) \in \widehat{G}_{0}$ may be realized as a unitary subrepresentation of $\iota \circ \alpha$.

Proof. The existence part of the theorem is handled by the Mackey bijection from Hig08, which is the map

$$
\mu: \iota(\sigma, \nu) \longmapsto \pi(\sigma, \nu) .
$$

According to Lemma 6.1.2, the representation $\pi(\sigma, \nu)$ of $G_{0}$ occurs within $\iota(\sigma, \nu) \circ \alpha$ with multiplicity equal to the multiplicity with which the weight $\sigma$ occurs in the representation of the compact connected group $K_{\nu}$ with highest weight $\sigma$; this latter multiplicity is 1 .

As for uniqueness, suppose we are given any bijection $\mu$, as in the statement of the theorem. It follows from Lemma 6.1.2 that $\mu$ must have the form

$$
\mu: \iota(\sigma, \nu) \longmapsto \pi(\theta, \nu)
$$

for some $\theta \in \widehat{M} / W_{\nu}$ with $[\theta] \geq[\sigma]$. So for each fixed $\nu \in \mathfrak{a}^{*}$, we obtain from $\mu$ a bijection of sets

defined by

$$
\mu_{\nu}: \widehat{M} / W_{\nu} \longrightarrow \widehat{M} / W_{\nu}
$$

$$
\mu_{\nu}: \iota([\sigma], \nu) \longmapsto \pi\left(\mu_{\nu}[\sigma], \nu\right) .
$$

Here our adjusted notation reflects the fact that $\iota(\sigma, \nu)$ and $\pi(\sigma, \nu)$ depend only on $[\sigma]$. We need to show that $\mu_{\nu}$ is the identity map, for all $\nu$.

Now it follows from Lemma 6.1.2 that $\mu_{\nu}$ has the property that

$$
\mu_{\nu}([\sigma]) \geq[\sigma] \quad \forall[\sigma] \in \widehat{M} / W_{\nu},
$$

and so of course the inverse bijection has the property that

$$
\mu_{\nu}^{-1}([\sigma]) \leq[\sigma] \quad \forall[\sigma] \in \widehat{M} / W_{\nu} .
$$

It follows from this that $\mu_{\nu}^{-1}$ maps each of the finite sets

$$
S_{\sigma}=\left\{[\theta] \in \widehat{M} / W_{\nu}:[\theta] \leq[\sigma]\right\}
$$

into itself, and this in turn implies that $\mu_{\nu}^{-1}$ is the identity map, as required.

\section{REFERENCES}

[AA19] A. Afgoustidis and A.-M. Aubert, Continuity of the Mackey-Higson bijection, Preprint, arXiv:1901.00144 (2019).

[Afg15] Alexandre Afgoustidis, On the analogy between real reductive groups and Cartan motion groups: contraction of irreducible tempered representations, Duke Math. J. 169 (2020), no. 5, 897-960, DOI 10.1215/00127094-2019-0071. MR4079418

[Afg16] Alexandre Afgoustidis, On the analogy between real reductive groups and Cartan motion groups: a proof of the Connes-Kasparov isomorphism, J. Funct. Anal. 277 (2019), no. 7, 2237-2258, DOI 10.1016/j.jfa.2019.02.023. MR.3989145

[BCH94] Paul Baum, Alain Connes, and Nigel Higson, Classifying space for proper actions and $K$-theory of group $C^{*}$-algebras, $C^{*}$-algebras: 1943-1993 (San Antonio, TX, 1993), Contemp. Math., vol. 167, Amer. Math. Soc., Providence, RI, 1994, pp. 240-291, DOI 10.1090/conm/167/1292018. MR/1292018 
[BK18] Alexander Braverman and David Kazhdan, Remarks on the asymptotic Hecke algebra, Lie groups, geometry, and representation theory, Progr. Math., vol. 326, Birkhäuser/Springer, Cham, 2018, pp. 91-108. MR3890206

[CCH16] Pierre Clare, Tyrone Crisp, and Nigel Higson, Parabolic induction and restriction via $C^{*}$-algebras and Hilbert $C^{*}$-modules, Compos. Math. 152 (2016), no. 6, 1286-1318, DOI 10.1112/S0010437X15007824. MR3518312

[CH90] Alain Connes and Nigel Higson, Déformations, morphismes asymptotiques et K-théorie bivariante (French, with English summary), C. R. Acad. Sci. Paris Sér. I Math. 311 (1990), no. 2, 101-106. MR1065438

[Dix77] Jacques Dixmier, $C^{*}$-algebras, North-Holland Publishing Co., Amsterdam-New YorkOxford, 1977. Translated from the French by Francis Jellett; North-Holland Mathematical Library, Vol. 15. MR0458185

[Ful84] William Fulton, Intersection theory, Ergebnisse der Mathematik und ihrer Grenzgebiete (3) [Results in Mathematics and Related Areas (3)], vol. 2, Springer-Verlag, Berlin, 1984. MR732620

[GfN50] I. M. Gel'fand and M. A. Nă̌mark, Unitarnye predstavleniya klassičeskih grupp (Russian), Trudy Mat. Inst. Steklov., vol. 36, Izdat. Nauk SSSR, Moscow-Leningrad, 1950. MR0046370

[HC54] Harish-Chandra, The Plancherel formula for complex semisimple Lie groups, Trans. Amer. Math. Soc. 76 (1954), 485-528, DOI 10.2307/1990793. MR63376

[Hig08] Nigel Higson, The Mackey analogy and K-theory, Group representations, ergodic theory, and mathematical physics: a tribute to George W. Mackey, Contemp. Math., vol. 449, Amer. Math. Soc., Providence, RI, 2008, pp. 149-172, DOI 10.1090/conm/449/08711. MR2391803

[Hig10] Nigel Higson, The tangent groupoid and the index theorem, Quanta of maths, Clay Math. Proc., vol. 11, Amer. Math. Soc., Providence, RI, 2010, pp. 241-256. MR 2732053

[HSSH18] Ahmad Reza Haj Saeedi Sadegh and Nigel Higson, Euler-like vector fields, deformation spaces and manifolds with filtered structure, Doc. Math. 23 (2018), 293-325. MR 3846057

[Kna02] Anthony W. Knapp, Lie groups beyond an introduction, 2nd ed., Progress in Mathematics, vol. 140, Birkhäuser Boston, Inc., Boston, MA, 2002. MR1920389

[Mac49] George W. Mackey, Imprimitivity for representations of locally compact groups. I, Proc. Nat. Acad. Sci. U.S.A. 35 (1949), 537-545, DOI 10.1073/pnas.35.9.537. MR31489

[Mac75] George W. Mackey, On the analogy between semisimple Lie groups and certain related semi-direct product groups, Lie groups and their representations (Proc. Summer School, Bolyai János Math. Soc., Budapest, 1971), Halsted, New York, 1975, pp. 339-363. MR0409726

[Mac76] George W. Mackey, The theory of unitary group representations, Chicago Lectures in Mathematics, University of Chicago Press, Chicago, Ill.-London, 1976. Based on notes by James M. G. Fell and David B. Lowdenslager of lectures given at the University of Chicago, Chicago, Ill., 1955. MR 0396826

[Ped79] Gert K. Pedersen, $C^{*}$-algebras and their automorphism groups, London Mathematical Society Monographs, vol. 14, Academic Press, Inc. [Harcourt Brace Jovanovich, Publishers], London-New York, 1979. MR548006

[PP83] M. G. Penington and R. J. Plymen, The Dirac operator and the principal series for complex semisimple Lie groups, J. Funct. Anal. 53 (1983), no. 3, 269-286, DOI 10.1016/0022-1236(83)90035-6. MR724030

[Rom19] Angel Roman-Martinez, Mackey Bijection for Some Reductive Groups and Continuous Fields of Reduced Group $C^{*}$-Algebras, ProQuest LLC, Ann Arbor, MI, 2019. Thesis (Ph.D.)-The Pennsylvania State University. MR 4132485

[Sol12] Maarten Solleveld, On the classification of irreducible representations of affine Hecke algebras with unequal parameters, Represent. Theory 16 (2012), 1-87, DOI 10.1090/S1088-4165-2012-00406-X. MR2869018

[Wal71] Nolan R. Wallach, Cyclic vectors and irreducibility for principal series representations, Trans. Amer. Math. Soc. 158 (1971), 107-113, DOI 10.2307/1995774. MR281844 
Department of Mathematics, Penn State University, University Park, Pennsylvania 16802

Department of Mathematics, Penn State University, University Park, Pennsylvania 16802

Current address: Department of Mathematics, William and Mary, Williamsburg, Virginia 23185

Email address: arroman@wm.edu 